\title{
Sliding Window used for Robustness and Fidelity Optimization Employing Neighborhood Concept and Genetic Algorithm with Tournament Selection
}

\author{
Sachin Goyal \\ Department of Information Technology \\ U.I.T, R.G.P.V, Bhopal
}

\author{
Roopam Gupta \\ Department of Information Technology \\ U.I.T, R.G.P.V, Bhopal
}

\begin{abstract}
Digital media security is the principal concern in the today's technological world. Due to easy production, transmission and dissemination of digital data, security threats occur while dealing with digital data. Digital watermarking is one of the growing and promising technologies to protect the digital data from being tampered and also widely used for copyright protection. Several watermarking techniques in spatial and frequency domain were given by various researchers which suffered from problems of poor robustness and fidelity. The evolutionary techniques using genetic algorithm provide an alternative way of creating watermarks with promising values of robustness and fidelity aspect of watermarking. This paper deals with design and development of a new watermarking technique which uses genetic algorithm to identify locations within the cover image for watermark insertion in spatial domain and then apply the average neighborhood concept for the purpose of watermark insertion and extraction ensuring higher fidelity and robustness and resilience to several possible image attacks. Genetic search often produces same watermark locations in different populations for watermark insertion resulting in poor value of fidelity and robustness, which need to be checked. Sliding window concept introduced in this paper uses a set of a few genes which are serially shuffled to get new set of locations for watermarking during each population generation and helps in enhancing robustness and fidelity aspect of watermarking. Tournament selection has been used while using the genetic algorithms developed in the paper.
\end{abstract}

\section{Keywords}

Digital watermarking, Fidelity, Robustness, Neighborhood concept, Sliding window.

\section{INTRODUCTION}

There are many watermarking methods [1] which have been developed to embed a watermark into the cover image. Some of the important contributions of various proposed methods of digital watermarking during initial research period in digital watermarking are presented here. A watermark was generated using the least significant bit of the original image by Schyndel et.al [2] to produce the watermarked image. The watermark was extracted from a suspected image by taking the least significant bits at the proper locations. Cross-correlation of the original and extracted watermark was made by Schyndel, which showed that the resulting image contained an invisible watermark with simple extraction procedures. Cox et al. [3] pointed that, in order for a watermark to be robust to attack, it must be placed in perceptually significant areas of the image. Yen and Huey et al [4] chose pair of position with same quantization scale in standard JPEG quantization table as cover image and it use the concept of discrete cosine Transformation. Huang at el[6] paper used a concept of DCT and proposed a Progressive watermarking Techniques with genetic algorithm. B.sikander at el [5] presents a new method for adaptive watermark strength optimization in discrete cosine transform domain. In this paper GA is used as to intelligently select watermark strength. Proposed method use genetic algorithm to optimize the strength of the watermark for mid band DCT coefficients. M.J Anwar et al [7] this method is more robust against JPEG compression. In this method genetic algorithm is used to find best position in image block that the positions have a mathematical relationship. Somying promcharoen and yuttapong rangsanseri[8] this approach used a fuzzy C-mean algorithm to classify $8 \times 8$ DCT block as texture or non-texture region. In this paper, a digital image watermarking by using genetic algorithm to optimize parameters used in block based DCT watermark embedding. M.Rafig[9] have proposed a watermarking algorithm in the DCT domain using an evolutionary algorithm. S.Goyal et al [10] use a roulette-wheel strategy in genetic algorithm and optimize the fidelity in spatial domain.

However, these techniques suffer from the problems of unsatisfactory values of fidelity and robustness to various attacks as discussed in these papers. This paper proposes a technique which employs genetic algorithm to find a suitable location for watermark insertion within a cover image using neighborhood concept for optimizing a fidelity and robustness.

Section II discusses the concept of genetic algorithm Section III discusses the Algorithm for optimization of Robustness and Fidelity using genetic algorithms. Section IV show Experimental Result and Conclusion is given in section V. 


\section{GENETIC ALGORITHM}

Genetic algorithms [11, 12] are search algorithms based on mechanics of natural selection and natural genetics. They combine survival of fittest among string structures with a structured yet randomized information exchange to form search algorithms with some of the innovative flair of human search. In every search, a new set of artificial creatures (strings) is created using bits and pieces of fittest of the old creatures. Conventional search techniques are not very suitable for optimizing non-linear functions with multiple variables. However, genetic algorithms this can be conveniently done. In the genetic algorithms, the parameters are represented by en encoded binary string called the "chromosome" and the elements in the binary strings or the "genes" are adjusted to maximize or minimize the fitness values. The fitness function has to be carefully selected specific to a particular application and the kind of optimization required. Thus, the entire process of genetic algorithm starts with a set of proposed solutions randomly generated and try to produce further possible solutions to achieve the desired optimization. This is the reason of the wide application of genetic algorithms in optimization areas.

The core components of the GA [11] are as under.

1. Fitness Value

2. Selection

3. Crossover

4. Mutation

\section{ALGORITHM OPTIMIZATION OF ROBUSTNESS AND FIDELITY WITH SLIDING WINDOW NEIGHBOURHOOD \\ CONCEPT}

Step 1: Let the cover image (cimage) used to embed the watermark be given as

cimage $=\left[c_{11}, c_{12}, \ldots \ldots \ldots \ldots c_{i j} \ldots \ldots \ldots c_{m c \times n c}\right]$

where $1<=i<=m c$ and $1<=j<=n c$

Step 2 : cimage is now reshaped into row vector containing $\mathrm{mc} * \mathrm{nc}$ entries

$\operatorname{cimage}((i-1) * n c+j)=\operatorname{cimage}(i, j)$

where, $1<=i<=m c$ and $1<=j<=n c$

This produces a row vector

$\operatorname{cimage}\left(c_{1}, c_{2}, \ldots \ldots \ldots c_{i}, \ldots \ldots \ldots c_{m c * n c}\right)$

The size of the cover image is given as $\mathrm{s}=\mathrm{mc}^{*} \mathrm{nc}$

Step 3: Let the watermark be given as

$w=\left[w_{11}, w_{12}, \ldots \ldots w_{i j}, \ldots \ldots w_{m n}\right]$ where $1<=i<=m$ and $1<=j<=n$

Step 4: Now the array fitness is initialized with all zeros.

fitness $=[0,0,0,0 \ldots$ pop_size times $]$

Step 5 : The array population is created randomly as

Population $=\left[p_{11}, p_{12}, \ldots \ldots p_{i j} \ldots, p_{\text {popsize }}\right.$ no_of_genes $]$

where $1<=i<=$ pop_size and $i<j<=n o \_o f \_g e n e s$ and $p_{i j}=0$ or 1 (randomly assigned)

Step 6 : Now, the watermark is converted into a single dimension vector $\mathrm{W}_{\mathrm{m} 1}$ as

$W_{m l}=\left[w_{1}, w_{2}, \ldots w_{i}, \ldots w_{m * n}\right]$

The total number of watermark locations is given by $m^{*} n$.

Step 7 : Now the individual chromosomes from the population are extracted .

chromosomes $[k]=\left[p_{k 1}, p_{k 2}, \ldots p_{k i}, \ldots . . p_{k n o \_o f \_g e n e s}\right]$

where, $1<=k<=$ pop_size and $p_{k l}, p_{k 2}, \ldots p_{k i}$, are the genes of the population.

Step 8: Now, the individual chromosomes are brought into variable range as following.

First of all, the watermark locations in the cover image $x(i)$ are found.

$x(i)=\sum$ chromosome $(y+j) \times 2^{\wedge}-j$

where $1<=i<=$ watermark_location and

$1<=j<=n o \_o f \_g e n e s /$ watermark_location, $y=0$

then

$x(i)=1+f i x(($ variable_range -1$) * x(i) /$

$$
\left.\left(1-2^{- \text {no_of_genes/watermark_location })}\right)\right)
$$

where $1<=i<=$ watermark_location

Step 9 : Now the Pixel intensity value of the cover image as modified according to average neighborhood pixel intensity.

Firstly calculate the average pixel intensity of selected location $x$ (i) by using neighborhood concept.

$$
\text { if cimage }(x(i))<\text { averagepixelintensity }
$$

new_cimage $(x(i))=$ averagepixelintensity $+E s$ 
where $1<=i<=$ watermark_location and $W_{m I}(i)=1$,

if cimage $(x(i))>$ averagepixelintensity

new_cimage $(x(i))=$ averagepixelintensit $-E s$

where $1<=i<=$ watermark_location and $W_{m l}(i)=0$

Es is embedding strength

Step 10: Now the similarity between the modified cover image and the original cover image is termed as fitness $\mathrm{f}$ calculated as per following equation[13]

$$
f(i)=10 * \log 10\left((\text { max_value })^{2} / \text { psnrsum }\right)
$$

where $1<=i<=p o p \_s i z e$ and

$\max \_v a l u e=\operatorname{Max}\left[\operatorname{cimage}\left(c_{1}, c_{2}, \ldots \ldots \ldots c_{m c} *_{n c}\right)\right]$

and psnrsum[13] is given by

$$
\begin{aligned}
& \text { psnrsum }= \\
& \left.\sum_{\mathrm{var}=1}^{m c^{*} n c}\left(n e w_{-} \text {cimage }(\mathrm{var})-\operatorname{cimage}(\operatorname{var})\right)^{\wedge} 2\right)
\end{aligned}
$$

Step 11: Now maxfitness and bestindividual index $k$ is derived

$$
\begin{aligned}
& \text { fitness }(i)<\text { fitness }(k) \\
& \forall i \therefore 1<=1<=\text { pop_size and } i<>k_{\text {s }} \text {, size }
\end{aligned}
$$

Step 12: Tournament selection is done, index I1 and I2 are selected.

$$
\begin{aligned}
& I 1=\text { itempl if } f(\text { itemp } 1)>(\text { itemp } 2) \\
& \text { itemp } 2 \text { iff(itemp2)>f(itemp } 1)
\end{aligned}
$$

where $r<$ tournamentselectionparameter and itemp1 and itemp2 are two randomly generated index such that.

$1<=$ itemp $1<=$ pop_size and $1<=$ itemp $2<=$ pop_size and $\mathrm{r}$ is randomly generated between $0<=r<1$.

$$
\begin{aligned}
& I 2=\text { itemp } 2 \text { if } f(\text { itemp } 1)>f(\text { itemp } 2) \\
& \text { itempl if } f(\text { itemp2) }>\text { f(itempl) }
\end{aligned}
$$

where $r>$ tournamentselectionparameter and itemp1 and itemp2 are two randomly generated index such that

$1<=$ itemp $1<=$ pop_size and $1<=$ itemp $2<=$ pop_size and $\mathrm{r}$ is randomly generated between $0<=r<1$.
The chromosome corresponding to the selected indices I1 and I2 are generated

$$
\begin{aligned}
& \text { chromosomel = population }(I 1, J) \\
& \text { where } 1<=J<=\text { no_of_genes }
\end{aligned}
$$

chromosome 2 = population $(I 2, J)$

$$
\text { where } 1<=J<=\text { no_of_genes }
$$

Step 13 : Now the crossoverpoint is selected randomly such that

$$
1<=\text { crossoverpoint }<\text { no_of_genes }
$$

Now crossover between chromosome 1 \& chromosome 2 is performed as per following equation and new chromosome pair called newChromosome_pair is generated

newChromosome_pair $(1, j)=$ chromosome $1(j)$
newChromosome_pair $(2, j)=$ chromosome $2(j)$

$$
\forall j \because, 1<=j<=\text { crossoverpoint }
$$

OR

newChromosome_pair $(1, j)=$ chromosome $2(j)$

newChromosome_pair $(2, j)=$ chromosomel $(j)$

$$
\forall j \because \text {, crossoverpoint }<j<=\text { no_of_genes }
$$

Step 14 : Now new_population is defined as

$$
\begin{aligned}
& \text { new_population }(i, j)=\text { newChromosome_pair }(1, j) \\
& \text { new_population }(i+1, j)=\text { newChromosome_pair }(2, j)
\end{aligned}
$$

$\forall j \because, 1<=j<=n o \_o f \_g e n e s$ and $r<$ crossoverprobabilty, where $r$ is random number generated between $0<=r<1$

If $r>$ crossoverprobability then crossover is not performed and new_population is given by

new_population $(i, j)=$ chromosome 1

new_population $(i+1, j)=$ chromosome 2

$$
\forall j \because, 1<=j<=\text { no_of_genes }
$$


Step 15 : Now the new_population is redefined such that each chromosome in the new_population is mutated depend on the mutation probability.

$$
\begin{aligned}
& n e w \_p o p u l a t i o n(i, j)=1-(\text { new_population }(i, j)) \\
& \forall i, j \because 1<=i<=\text { pop_size and } 1<=j<=n o \_o f \_g e n e s \text { and }
\end{aligned}
$$
$r<$ mutation_probability, where $r$ is random number generated between $0<=r<1$

Step $16:$ gen_count $=$ gen_count +1

Step $17:$ population= new_population

Repeat all step 7 to 16 for gen_count $<=$ no_of_generation

Now the maxfitness and bestwatermark location is obtained.

Table-I Genetic Algorithm Parameters

\begin{tabular}{|l|l|}
\hline Parameters & Values \\
\hline Generation & $30-50$ \\
\hline Population Size & 6 \\
\hline $\begin{array}{l}\text { Tournament } \\
\text { Selection rate }\end{array}$ & 0.90 \\
\hline $\begin{array}{l}\text { Crossover } \\
\text { Probability }\end{array}$ & 0.90 \\
\hline Mutation rate & 0.025 \\
\hline Window Size & 3 \\
\hline
\end{tabular}

\subsection{Neighborhood concept}

In this concept, first of all a suitable location is selected by genetic algorithm for watermark insertion. Now, for the purpose of insertion of watermark, the neighborhood of the concerned location is utilized. A neighborhood of $3 * 3$ pixels is chosen surrounding the concerned pixel and the average of the pixel intensity values of the neighborhood pixel is compared with the pixel intensity where the watermark has to be inserted. If the pixel intensity value is higher than this average a binary 1 is inserted else a binary 0 is inserted in the concerned pixel.

There are two Case occur when be inserted watermark bit in to cover image.

In first case, when watermark mark bit 1 is inserted if the value of selected location pixel is less then average intensity value then increase the intensity of selected location according to equation(30).

$$
\mathrm{SL}=\mathrm{API}+\mathrm{Es}
$$

In second case when watermark bit 0 is inserted if the value of selected location pixel is grater then average intensity value then decrease the value of selected location according to equation (31)

$$
\mathrm{SL}=\mathrm{API}-\mathrm{Es}
$$

Where SL- Selected location, API-Average Pixel Intensity and Es embedding strengths

The advantage of using neighborhood concept for watermark is reflected in terms of higher robustness. This is due to the reason that during image attacks, the average of surrounding pixels is not significantly affected and thus it prevents the watermark bit from getting modified.

\subsection{Sliding window concept}

During watermarking with genetic algorithms, there is always a possibility of getting the similar locations during genetic search for watermark insertion. To prevent this from happening, sliding window concept is being utilized in the paper. In this concept, a set of 3 genes is selected to encode a watermark location (say from 1-3and then this set is shifted right by 1 gene (say from 24) for the next watermark selection. The repeated use of this technique ensures different watermark locations to be selected and thus avoiding the limitation and probability of finding same locations in the search space. This results in enhanced robustness and fidelity as indicated by various experiments.

\section{EXPERIMENTAL RESULTS}

Tournament selection strategy is used to find out the desired locations for watermark insertion for Robustness and fidelity optimization. In our experiments, the cover images used is Lena size $(512 \times 512)$ and the binary two dimensional matrix with varying length in different experiments as the watermark and compare our result with other technique shown in table. Table I represents the various parameter setting used in the experiments for genetic algorithm. The PSNR value for Lena under different payload shown in Table II and Table III show value of NC under different type of attack.(i.e. Median filter, Mean filter, Sharpening, Salt \& Pepper, Gaussian attack). 
Table II Variation of PSNR with payload

\begin{tabular}{|l|l|l|}
\hline S.No. & Payload(bits) & PSNR(dB) \\
\hline 1 & 512 & 67.5563 \\
\hline 2 & 1024 & 62.9431 \\
\hline 3 & 2048 & 59.0142 \\
\hline 4 & 4096 & 55.6017 \\
\hline 5 & 16384 & 49.4107 \\
\hline
\end{tabular}

Table-III Variation of NC with Payload

\begin{tabular}{|c|c|c|c|c|c|}
\hline $\begin{array}{l}\text { S. } \\
\text { No }\end{array}$ & $\begin{array}{l}\begin{array}{l}\text { Payl } \\
\text { oad }\end{array} \\
\text { (bits) }\end{array}$ & $\begin{array}{l}\begin{array}{l}\text { Media } \\
\mathrm{n}\end{array} \\
\text { Filter } \\
\text { Attack }\end{array}$ & $\begin{array}{l}\text { Mean } \\
\text { Filter } \\
\text { Attack }\end{array}$ & $\begin{array}{l}\text { Salt \& } \\
\text { Peppe } \\
\text { r } \\
\text { Attack }\end{array}$ & $\begin{array}{l}\text { Sharpen } \\
\text { ing } \\
\text { Attack }\end{array}$ \\
\hline 1 & 512 & 1 & 1 & 1 & 1 \\
\hline 2 & 1024 & $\begin{array}{l}0.998 \\
9\end{array}$ & 0.9940 & $\begin{array}{l}0.990 \\
6\end{array}$ & 0.9719 \\
\hline 3 & 2048 & $\begin{array}{l}0.987 \\
2\end{array}$ & 0.9825 & $\begin{array}{l}0.989 \\
2\end{array}$ & 0.9652 \\
\hline 4 & 4096 & $\begin{array}{l}0.981 \\
9\end{array}$ & 0.9728 & $\begin{array}{l}0.985 \\
0\end{array}$ & 0.9484 \\
\hline 5 & $\begin{array}{l}1638 \\
4\end{array}$ & $\begin{array}{l}0.905 \\
1\end{array}$ & 0.9127 & $\begin{array}{l}0.952 \\
1\end{array}$ & 0.9140 \\
\hline
\end{tabular}

Table IV Comparison of Fidelity method with other method

\begin{tabular}{|l|l|l|l|l|l|}
\hline S.No. & Method & Image & Size & Watermark & PSNR \\
& $\begin{array}{l}\text { Method } \\
\text { bits }\end{array}$ & $(\mathrm{dB})$ \\
\hline 1 & Rafigh & Lena & $512 \times 512$ & 4096 & 49.74 \\
\hline 3 & Sothod[9] & & & & \\
\hline 4 & Sikandar & Lena & $512 \times 512$ & - & 35.6017 \\
\hline & Method[5] & & & 4096 & \\
\hline
\end{tabular}




\section{CONCLUSION}

In this paper a new sliding window with neighborhood concept is used for finding a suitable location in spatial domain. The Sliding window concept for serial shuffling of a set of genes has helped in producing different watermark locations. The results shown in this paper meets in the objectives in term of providing a satisfactory value of robustness and fidelity. Finally we compare our results with the other previous method. In future we optimize the robustness while keeping the reasonable value of fidelity and vice-versa.

\section{REFERENCES}

[1] SachinGoyal, R.Gupta, A.Bansal “ Survey of Digital Watermarking with Genetic Algorithm" in Computer Society of India Communication Jan-2010.pp22-25.

[2] R.G.Van Schyndel,A.Z.Tirkel and CF.Osborene, "A Digital Watermark" in Proc. IEEE International Conf. Image processing, 1994,vol.2 pp 86-92.

[3] J.Cox,J.Kilian, "A Secure Robust Watermark for Multimedia" in Proc. First International Workshop, vol 1174 of Lecture notes in computer science, pp. 185-206.

[4] Yen, Shwu Huey, et al. " A Watermarking Technique based on JPEG quantization table." Taipei, Taiwan: Proceeding of the 2006 International Computer Symposium (ICS2006).

[5] B.Sikander, M. Iahiaq, M.A. Jaffer,M.Tariq, A. Mirza "Adaptive digital watermarking of images using Genetic Algorithm" in Proc IEEE.
[6] Huang ,Pan, Shieh and Wang "Progressive watermarking techniques with genetic Algorithms" IEEE, 2003.

[7] M.J. Anwar, M.Ishtiaq, M.Iqqbal, M,Jaffar “ Blocked-based digital image watermarking using Genetic Algorithm" in Proc IEEE international conference on Emerging Technologies(ICET) 2010. pp 204-208.

[8] Somying Promchareon and Yuttapong Rangsanseri. “ Genetic watermarking with Block-Based DCT Clustering in IEEE International Symposium on Communication and Information Technologies(ISCIT-2008). Pp 346-351

[9] M.rafigh and M.Ebrahimi “ A Robust Evolutionary Based Digital Image Watermarking Technique in DCT Domain" in Proc IEEE Seventh International Conference on Computer Graphics, Imaging and Visualization 2010.pp 105-109.

[10] Sachin Goyal and Roopam Gupta "Optimization of fidelity with Adaptive genetic watermarking algorithm using roulette-wheel" in proc IEEE International conf. On Computational Intelligence Communication Networls", 2010. pp 591-596.

[11] Goldberg, D.E, "Genetic Algorithm in Search, Optimization \& machine learning", Addison-Weseley, 1989.

[12] M.Srinivas and Lalit M.Patnaik, "Genetic Algorithm: A Survey" IEEE, 1994.

[13] I.J. Cox, M.L Miller, J.A Bloom "Digital watermarking” Morgam Caufmann. 